\title{
Pirimidine derivatives as hepatoprotective agents
}

\author{
A.B. Vyshtakalyuk*, N.G. Nazarov, V.V. Zobov, V.E. Semenov, I.V. Galyametdinova, \\ G.V. Tcherepnev and V.S. Reznic
}

A.E. Arbuzov Institute of Organic and Physical Chemisrty Kazan Scientific Centre Russian Academy of Sciences, Kazan, Russia

*Corresponding author. E-mail: alex.vysh@mail.ru

\begin{abstract}
BACKGROUND: Research and development of effective hepatoprotective medicines is one of the priority areas of research in Russia. Literature data shows that active research and development of hepatoprotectors is carried out both in Russian and other countries [1-6]. Pirimidines are used as hepatoprotective medicines stimulating protein synthesis and reparation of hepatocytes in toxical and infectious liver disorders [7]. In our previous work ee have shown hepatoprotective properties of pyrimidine derivative, named Xymedon [8]. This research, funded by the Russian Science Foundation, is aimed at identifying the most effective hepatoprotectors among pirimidine derivatives.
\end{abstract}

OBJECTIVE: To test hepatoprotective properties of one of the new Xymedon (Xym) derivative L-ascorbate 1-(2-hydroxyethyl)-4,6-dimethyl-1,2-dihydro-pirimidine-2-one (Asc-Xym) on the toxic liver damage model induce by carbon tetrachloride $\left(\mathrm{CTC}, \mathrm{CCl}_{4}\right)$.

METHODS: The toxic liver damage in rats was modeled by subcutaneous injection of $\mathrm{CTC}\left(\mathrm{CCl}_{4}\right)$ in vegetable oil (mixed at 1:1 ratio) at a dose of $2 \mathrm{ml}$ per $\mathrm{kg}$. The experiments were carried out under two schemes: 1) oral administration of Xym or Asc-Xym preparations by gavage at the doses of 10 and $20 \mathrm{mg} / \mathrm{kg}$ followed by subcutaneous injection of CTC 1 hour after pyrimidine oral administration and continued for 3-4 days; - this was the design of preventive pyrimidine use, 2) liver damage modeling by CTC subcutaneous injections for 3 days followed by oral administration of Xym, Asc-Xym or Thiotriazolin (Thi) preparations at the doses of $20 \mathrm{mg} / \mathrm{kg}$ for 5 days; - this was the design of therapeutic scheme. The rats of control groups were injected with CTC according to the same schemes, but did not get any preparations. We looked at some biochemical parameters of blood serum: alanine aminotransferase (AlAT), aspartate aminotransferase (AsAT), their ratio (de Rytis coefficient), and the total protein level as the markers of toxic liver damage. We performed statistical data analysis by rank nonparametric Mann-Whitney U-criterion for comparison of two independent groups. We evaluated pathomorphologic characteristics of liver damage on the histological slices colored with hematoxylin and eosin.

RESULTS: Carbon tetrachloride (CTC) caused profound changes in the studied biochemical parameters of rats' blood serum. The AlAT activity level in the serum of control animals in the preventive scheme was 116,23 (the median) with the lower quartile and the upper quartile of 76,96 and 211,71 U/1 respectively; the AsAT level was 230,08/201,49-290,03 U/1; this was the increase in comparison with the reference values. De Rytis coefficient was $1,76 / 1,47-2,67$. This was the decrease in comparison with the reference values of intact group (36,37/28,18-43,3 U/1; 132,95/118,24-164,00 U/1 and 4,26/3,035,23 respectively). The differences were statistically significant at $P<0,001$. In the experimental groups the changes of the biochemical parameters with respect to the reference values were less marked than 
in Control. The AlAT level was 89,86/87,06-165,15; 103,23/38,19-270,87 U/1; 80,28/6,12-141,82 and $100,33 / 62,24-144,64 \mathrm{U} / 1$ in the groups of rats treated with Xym at the doses of 10 and $20 \mathrm{mg} / \mathrm{kg}$ or AscXym at the doses of 10 and $20 \mathrm{mg} / \mathrm{kg}$ respectively. Similarly, in the same groups the AsAT level was $211,19 / 170,20-250,16 ; 193,61 / 181,57-274,69 \mathrm{U} / 1 ; 190,91 / 65,21-198,65$ and $173,25 / 135,50-210,70 \mathrm{U} / 1$ respectively. The differences of the AsAT level were statistically significant at $P<0,05$ in comparison with Control in the both groups treated with Asc-Xym.

Nnearly 2 times increase of the AlAT level $(67,60 / 1,22-94,60 \mathrm{U} / 1)(P=0,00002)$ was shown in comparison with the reference values in the rats of Control group in the therapeutic scheme. However the AsAT level $(163,80 / 130,1-178,8 \mathrm{U} / 1)$ was only slightly higher than reference values. De Rytis coefficient $(2,07 / 1,78-3,48)$ was significantly lower than the reference values $(P=0,001)$. The total protein level $(59,36 / 55,17-60,10 \mathrm{~g} / \mathrm{l})$ was lower than the reference values $(65,06 / 62,06-68,98 \mathrm{~g} / \mathrm{l})$ by $8,4 \%$. The differences of biochemical parameters as compared with the reference values in rats of experimental groups treated with Xym, Thi and Asc-Xym at the doses $20 \mathrm{mg} / \mathrm{kg}$ were less than those in the Control groups. They were: AlAT 52,49/44,64-62,30 and 61,42/53,20-96,66 U/1, AsAT 105,00/94,7142,3 and $235,35 / 111,7-335,6 \mathrm{U} / 1$, de Rytis coefficient 2,09/1,87-2,28 and 3,24/1,86-4,53, total protein $63,10 / 62,46-64,27$ and $62,46 / 58,70-64,43 \mathrm{~g} / 1$ respectively in the groups treated with Xym and Thi. The values of the studied biochemical parameters AlAT $(39,04 / 32,46-44,24$ U/1), AsAT $(111,9 / 105,27-155$ U/1), de Rytis coefficient $(2,87 / 2,72-3,30)$, total protein $(62,89 / 61,46-68,14 \mathrm{~g} / 1)$ of the rats, treated with Asc-Xym, were the most close to the reference values in comparison with other experimental groups.

The analysis of histological slices revealed large areas of steatosis and necrosis of hepatocytes in Control groups in both schemes. These were less pronounced in experimental groups than in Control groups and particularly in rats, treated with Asc-Xym.

CONCLUSIONS: Hepatoprtotective properties of the new compound L-ascorbate 1-(2-hydroxyethyl)4,6-dimethyl-1,2-dihydropirimidine-2-one were established. The hepatoprotective efficacy of the compound is higher than that of Xymedon and Thiotriazolin.

Keywords: Rats, pyrimidine, liver damage, carbon tetrachloride, biochemical markers

\section{The research was financed by the Russian Science Foundation grant number 14-50-00014.}

\section{References}

[1] Vengerovskii AI, Baturina NO, Chuchalin VS, Saratikov AS. Effect of polyphenol-containing hepatoprotectors on the experimental chronic hepatitis pattern. Pharmaceutical Chemistry Journal. 1996;30(2):69-71. doi: 10.1007/BF02218867

[2] Kabankin AS, Gabrielyan LI. Relationship between structure and hepatoprotector activity of adamantine derivatives. Pharmaceutical Chemistry Journal. 2005; 39(3):135-9. doi: 10.1007/s11094-005-0102-3

[3] Shilova IV, Zhavoronok TV, Souslov NI, Novozheeva TP, Mustafin RN, Losseva AM. Hepatoprotective properties of fractions from meadowsweet extract during experimental toxic hepatitis. Bulletin of Experimental Biology and Medicine. 2008;146(7):54-7. doi: 10.1007/s10517-008-0226-Z

[4] Kushnerova NF, Sprygin VG, Rachmanin YuA. [Influence of complex plant polyphenol preparation Eklikit on process of liver functions recovery after alcohol intoxication]. Biomedical Chemistry. 2004;50(6):605-11. Russian

[5] Demirdag K, Bahcecioglu IH, Ozercan IH, Ozden M, Yilmaz S, Kalkan A. Role of L-carnitine in the prevention of acute liver damage induced by carbon tetrachloride in rats. Journal of Gastroenterology and Hepatology. 2004;19:333-8. doi: $10.1111 / j .1440-1746.2003 .03291 . x$

[6] Saratikov AS, Chuchalin VS, Ratkin AV, Ratkin EV, Fedoreev SA, Bulgakov VP. [Hepatoprotective properties of polyphenolic complexes from wood and cell culture Maak Amur]. Experimental and clinical pharmacology. 2005; 68(2):51-4. Russian

[7] Zobov VV, Nazarov NG, Vyshtakalyuk AB, Galyametdinova IV, Semenov VE, Reznik VS. Efficiency of new pirimidine derivatives influence on physical working capacity of rats in the test "swimming to failure". Human ecology. 2015;1:2835. Russian

[8] Vyshtakaliuk AB, Nazarov NG, Porfiriev AG, Zueva IV, Minnechanova OA, Mayatina OV, Reznik VS, Zobov VV, and Nicolskyi EE. The Influence of the Xymedon Preparation (Hydroxyethyldimethyldihydropyrimidine) on the Rat Liver Recovery under Toxic Damage Induced by Carbon Tetrachloride. Doklady Biochemistry and Biophysics. 2015;462(1):1436. doi: $10.1134 / \mathrm{S} 1607672915030011$ 